\title{
The interaction between the vastus medialis and vastus intermedius and its influence on the extensor apparatus of the knee joint
}

\author{
Karl Grob $^{1} \cdot$ Mirjana Manestar ${ }^{2} \cdot$ Luis Filgueira $^{3} \cdot$ Markus S. Kuster $^{4}$. \\ Helen Gilbey ${ }^{5}$ Timothy Ackland ${ }^{4}$
}

\begin{abstract}
Purpose Although the vastus medialis (VM) is closely associated with the vastus intermedius (VI), there is a lack of data regarding their functional relationship. The purpose of this study was to investigate the anatomical interaction between the VM and VI with regard to their origins, insertions, innervation and function within the extensor apparatus of the knee joint.

Methods Eighteen human cadaveric lower limbs were investigated using macro-dissection techniques. Six limbs were cut transversely in the middle third of the thigh. The mode of origin, insertion and nerve supply of the extensor apparatus of the knee joint were studied. The architecture of the VM and VI was examined in detail, as was their anatomical interaction and connective tissue linkage to the adjacent anatomical structures.

Results The VM originated medially from a broad hammock-like structure. The attachment site of the VM always
\end{abstract}

Electronic supplementary material The online version of this article (doi:10.1007/s00167-016-4396-3) contains supplementary material

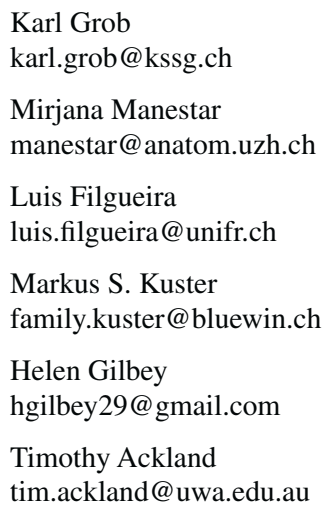

spanned over a long distance between: (1) patella, (2) rectus femoris tendon and (3) aponeurosis of the VI, with the insertion into the VI being the largest. VM units were inserted twice-once on the anterior and once on the posterior side of the VI. The VI consists of a complex multilayered structure. The layers of the medial VI aponeurosis fused with the aponeuroses of the tensor vastus intermedius and vastus lateralis. Together, they form the two-layered intermediate layer of the quadriceps tendon. The VM and medial parts of the VI were innervated by the same medial division of the femoral nerve.

Conclusion The VM consists of multiple muscle units inserting into the entire VI. Together, they build a potential functional muscular complex. Therefore, the VM acts as an indirect extensor of the knee joint regulating and adjusting the length of the extensor apparatus throughout the entire range of motion. It is of clinical importance that, besides the VM, substantial parts of the VI directly contribute to the medial pull on the patella and help to maintain medial tracking of the patella during knee extension. The interaction between the VM and VI, with responsibility for the

Department of Orthopaedic Surgery, Kantonsspital St. Gallen, Rorschacher Strasse 95, 9007 St. Gallen, Switzerland

2 Department of Anatomy, University of Zürich-Irchel, Winterthurerstrasse 190, 8057 Zurich, Switzerland

3 Department of Anatomy, University of Fribourg, Rue Albert Gockel 1, 1700 Fribourg, Switzerland

4 The University of Western Australia, 35 Stirling Highway, Crawley, Perth, WA 6009, Australia

5 Hollywood Functional Rehabilitation Clinic, Perth, WA, Australia 
extension of the knee joint and influence on the patellofemoral function, leads readily to an understanding of common clinical problems found at the knee joint as it attempts to meet contradictory demands for both mobility and stability. Surgery or trauma in the anteromedial aspect of the quadriceps muscle group might alter a delicate interplay between the VM and VI. This would affect the extensor apparatus as a whole.

Keywords Vastus medialis · Vastus intermedius - Extensor apparatus of the knee joint - Quadriceps muscle group ·

Extensor mechanism of the knee joint

\section{Introduction}

The vastus medialis (VM) and vastus intermedius (VI) muscles are part of the extensor apparatus of the knee joint. Both muscles are situated close to each other on the anterior and medial aspect of the femur. The VM and VI work together with the other components of the quadriceps muscle group (rectus femoris, vastus lateralis and tensor vastus intermedius [10] to produce knee extension torque when knee joint action is performed [43]. However, anatomical properties such as muscle volume, origin of the muscle and fibre-type composition seem to be inconsistent among the components of this muscle group [1]. These variations may be attributed to different functional roles or contributions among synergists of the extensor apparatus of the knee joint.

There is a large quantity of the literature regarding the importance of the VM as a medial stabilizer of the femoropatellar joint [19, 21, 26, 33, 49, 51, 53]. In contrast, very little has been written about the VI and its anatomical interaction with the VM. Traditionally, the VM is described as largely separated from the anterior and the medial side of the femur by the VI. It arises from the lower portion of the intertrochanteric line, spiral line, medial lip of the linea aspera, upper part of the medial supracondylar line, the tendons of the adductor longus and magnus, and the medial intermuscular septum. It has been proposed that the fibres of VM pass downwards and forward and end in the common tendon of the quadriceps muscle, which inserts into the patella and becomes connected with the capsule of the knee joint [37, 39, 44, 52]. In addition to this classic description, great variation in the distal part of the VM has been found [19, 26, 46, 49, 51, 53].

According to anatomy textbooks, the VI arises from the anterior and lateral surfaces of the upper two-thirds of the femoral shaft, and from the lower part of the lateral intermuscular septum [43]. The VI inserts into the posterior surface of the upper border of the patella, posterior to the
VM, through tendon fibres that make up the deep part of the quadriceps tendon [17, 41].

Although the VM and VI course are adjacent to each other, there is a lack of data regarding their functional relationship or interaction. According to many textbooks, atlases of anatomy and journals, the VI and VM are separated by an intermuscular space or septum [17, 35, 37, 39, 41]. However, previous anatomical dissections revealed that the interaction between the components of the quadriceps muscle group is more complex and intricate than was initially described. Furthermore, dissections revealed different morphological findings between the VI and VM compared to classic descriptions in the literature $[10,12,13]$. Loss of knee extension occurs most rapidly due to inactivity of the VM (28). Patellofemoral pain syndrome is thought to be associated with lateral malalignment of the patella [28]. Possible causes of this malalignment include a hypotrophy or atrophy of the VM, which might affect the VI and other components of the quadriceps muscle group indirectly. The purpose of this study was to investigate the anatomical connection between the VI and VM, as well as their innervations, origins, insertions and contributions to the function of the extensor apparatus of the knee joint.

\section{Materials and methods}

Eighteen cadaveric lower limbs from 12 specimens (6 paired and 6 unpaired), from eight males and four females, with a mean age at death of 77 years (range $=67-86$ years), were investigated using macro-dissection techniques. The cadaver parts were obtained from the institutional body donation programme (http://www.anatom.uzh.ch/Bodydonation.html) following the ethical guidelines "On the use of cadavers and parts of cadavers in medical research and for pre-, postgrad and continued education and research with human subjects" by the Academy of Medical Sciences (SAMS). All lower limbs were embalmed in a formalinbased solution. The thighs were examined by a standardized dissection protocol. Each lower limb was placed supine on a dissection table, and an anterior approach to the hip joint was performed. Resection of the joint capsule followed, and the proximal border of the muscle bellies of the quadriceps muscle group was localized. After an additional incision proximal to the inguinal ligament, the femoral nerve was dissected and traced distally. All the muscle branches of the femoral nerve to the sartorius and the different components of the quadriceps muscle were identified. Finally, the sartorius and the rectus femoris muscles were transected distally and elevated proximally. All nerve branches to the VI and VM were carefully dissected. The saphenous nerve was traced separately until it exited the 
adductor canal. VM was released from its medial attachment to the intermuscular septum. Six limbs from four specimens (two paired and two unpaired) were cut transversely in the middle third of the thigh. The origins, insertions, nerve supply and the architecture of the VM and VI were examined. Attention was paid to the anatomical interaction between the VM and VI, as well as their connective tissue linkage to the adjacent anatomical structures.

\section{Results}

\section{Origin, course and insertion of the vastus medialis}

The VM originated medially and dorsally from a broad hammock-like structure composed of the medial intermuscular septum and its great expansion between the distal continuation of the intertrochanteric line, the spiral line, the medial lip of the linea aspera down to the proximal part of the medial supracondylar line, the tendon of the adductor magnus, the adductor canal, the aponeurosis of adductor longus and the periarterial connective tissue of the groove for the femoral vessels (Fig. 1). The medial aponeurosis of the VM was always tightly bound to the aponeurosis of the adductors.

The insertion of the VM always spanned over a long distance in three sections (Figs. 1, 2, 3), being:

1. Section 1-The entire aponeurosis of the VI;

2. Section 2-The distal tendon of the rectus femoris; and

3. Section 3-The medial border of the patella and joint capsule.

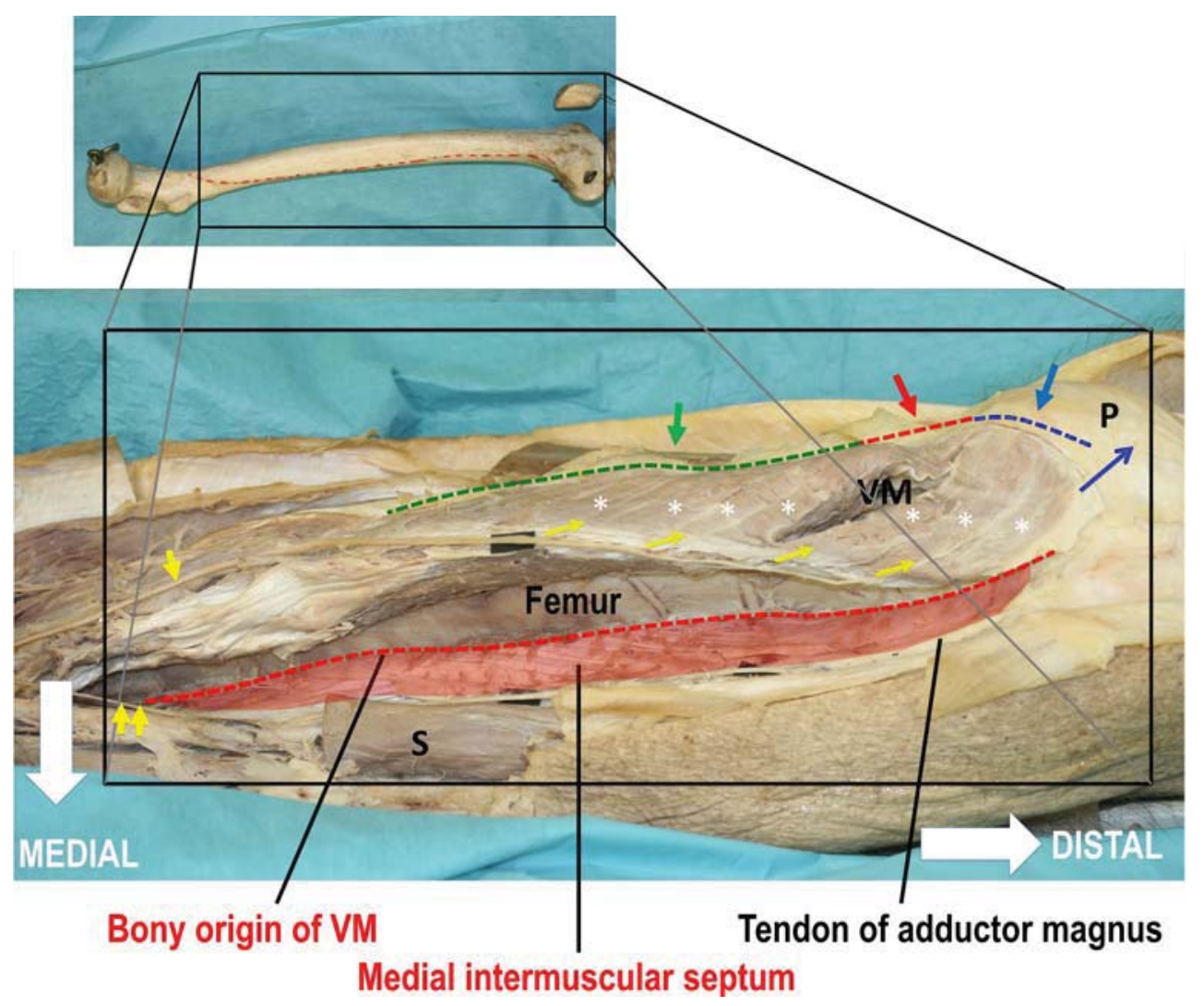

Fig. 1 Origin and insertion of the vastus medialis. Medial view of the distal two-thirds of a left thigh in slight external rotation is shown. The vastus medialis (VM) is lifted from its hammock-like origin (red transparent surface) and medial lip of the linea aspera and medial supracondylar line (long red dotted line). See also the corresponding femur (on the top) with the marked bony origin of the VM. The insertions of the VM into the patella $(\mathrm{P})$, the quadriceps tendon and the vastus intermedius are marked with blue, red and green dotted lines and arrows. Below the lifted VM, the medial surface of the femur is bare of muscle fibres and, therefore, not an attachment site for the
VM. This indicates the function of the medial femur as a gliding surface for the contracting VM muscle units $(*)$. The long nerve branch to the VM (single yellow arrow) courses distally along the anteromedial border of the muscle and remains, in contrast to the saphenous nerve (yellow double arrows), lateral to its superficial aponeurosis in a separate fibrotic tunnel. The nerve branch to the VM becomes gradually thinner as it gives up many side branches (thin yellow arrows) to and between the muscle units $(*)$ of the VM. Sections of the nerves are underlined with black paper for better visualization. The sartorius muscle $(\mathrm{S})$ is transected showing the underlying femoral artery 


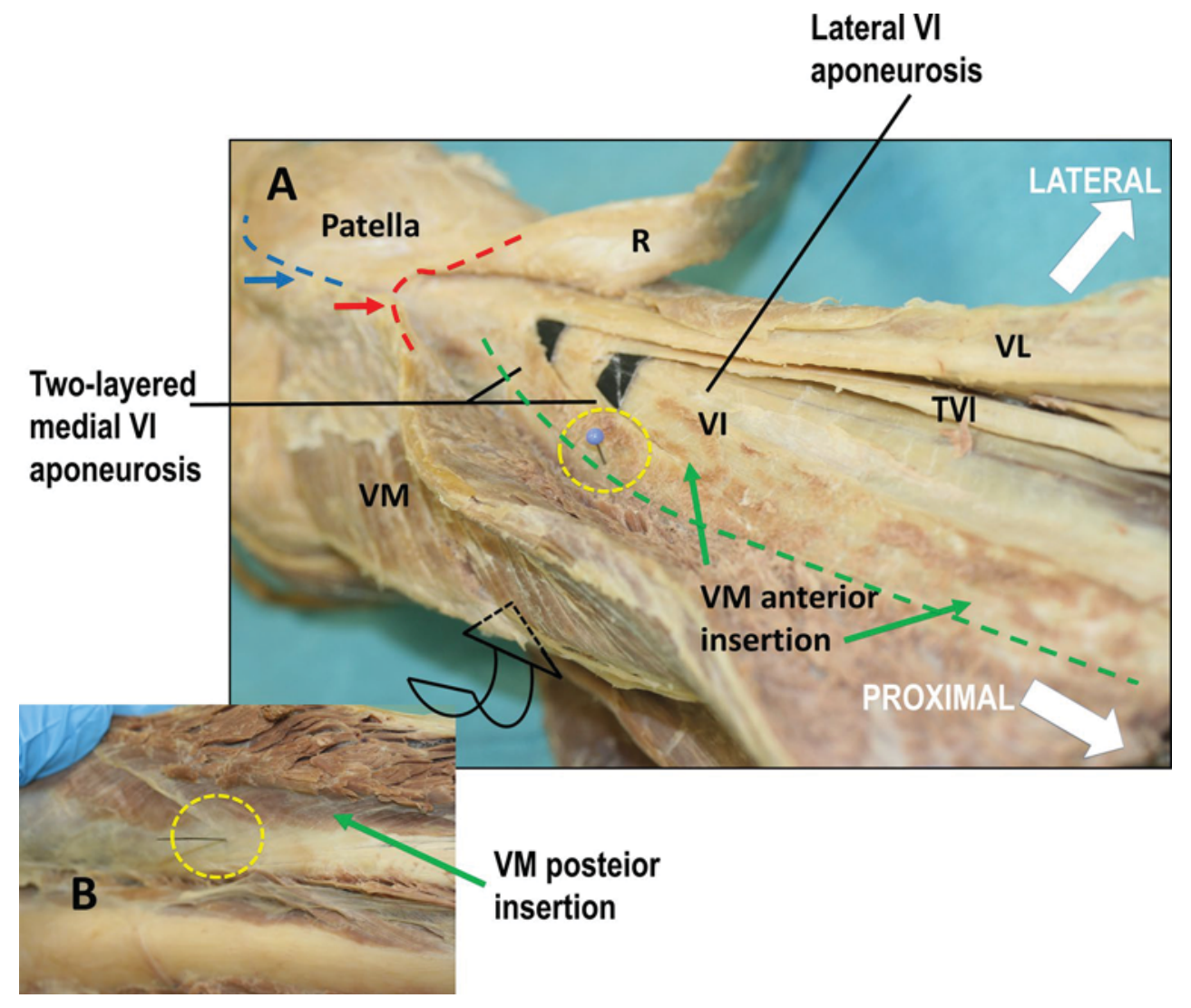

Fig. 2 Anterior and posterior insertion of the vastus medialis into the aponeurosis of the vastus intermedius. a The distal right thigh proximal to the knee joint is shown. The vastus medialis (VM) is released from its anterior insertion into the vastus intermedius (VI) and rectus femoris $(\mathrm{R})$ freeing the view to the multi-layered quadriceps tendon. The insertions of the VM into the patella $(\mathrm{P})$, the distal tendon of the rectus femoris $(\mathrm{R})$ reflected laterally and the vastus intermedius (VI) are marked with blue, red and green dotted lines and arrows. The green arrows indicate the anterior insertion of the VM (VM anterior)

The first section of this VM insertion was the largest and expanded over the whole length of the VI aponeurosis. It had a fleshy double insertion-a strong and large insertion on the anterior side and a weaker insertion on the posterior side of the VI aponeurosis. VM clamps the VI aponeurosis like a clip holding a sheet of paper (clip-type double insertion) (Figs. 2, 3, 4). This part of the VI insertion is broad and strong especially on its anterior side (Figs. 2, 3). Distally, as more muscle fibres of the VM blended into the VI aponeurosis, its medial part divided into deep and superficial layers. These two layers finally crossed over the lateral part of the VI aponeurosis (Figs. 2, 3).

The second section of VM insertion occurs on the medial edge of the most distal $52 \mathrm{~mm}$ of the rectus femoris tendon (range $=40-61 \mathrm{~mm}, \mathrm{SD}=0.7$ ) where the $\mathrm{VM}$ blends its fibres on its upper and lower side (Figs. 2, 3). Distally, this attachment continues into the third section of the VM insertion at the medial border of the patella. An

into the aponeurosis of VI. Vastus lateralis (VL) and tensor vastus intermedius (TVI) are also labelled. The fusing points of the different layers of the quadriceps tendon are underlined with black paper for better visualization. The blue headed needle perforates the medial VI aponeurosis (see the exit point of the needle on $\mathbf{b}$ ). $\mathbf{b}$ The VM is lifted laterally showing the femoral side of the VI aponeurosis with the posterior inserting VM muscle fibres (green arrow, VM posterior). The medial femur is bare of muscle fibres and not an attachment site for the VM

aponeurotic expansion of this section reinforces the capsule of the knee joint (Figs. 2, 3). Proximally, the muscle fibres of VM are aligned almost longitudinally to the axis of the femoral shaft. Distally, the fibres run in an oblique direction and near the end are almost transverse in orientation. The VM consists of multiple muscle units, which become more oblique in direction from proximal to distal (Fig. 1).

The medial surface of the shaft of the femur is bare and overstretched once by the medial part of the VI aponeurosis and once by the inserting muscle fibres of VM (Figs. 1, $2 b)$. The VM units, arising from the hammock-like origin (as described above), are fleshy except for their lowest part, where they run over the medial condyle of the femur and the thin muscle sheet of the articularis genus. Here, the VM consists of a gliding aponeurosis that generally merges with the superficial layer of the medial VI aponeurosis and laterally from the aponeurosis of the vastus lateralis. Both 


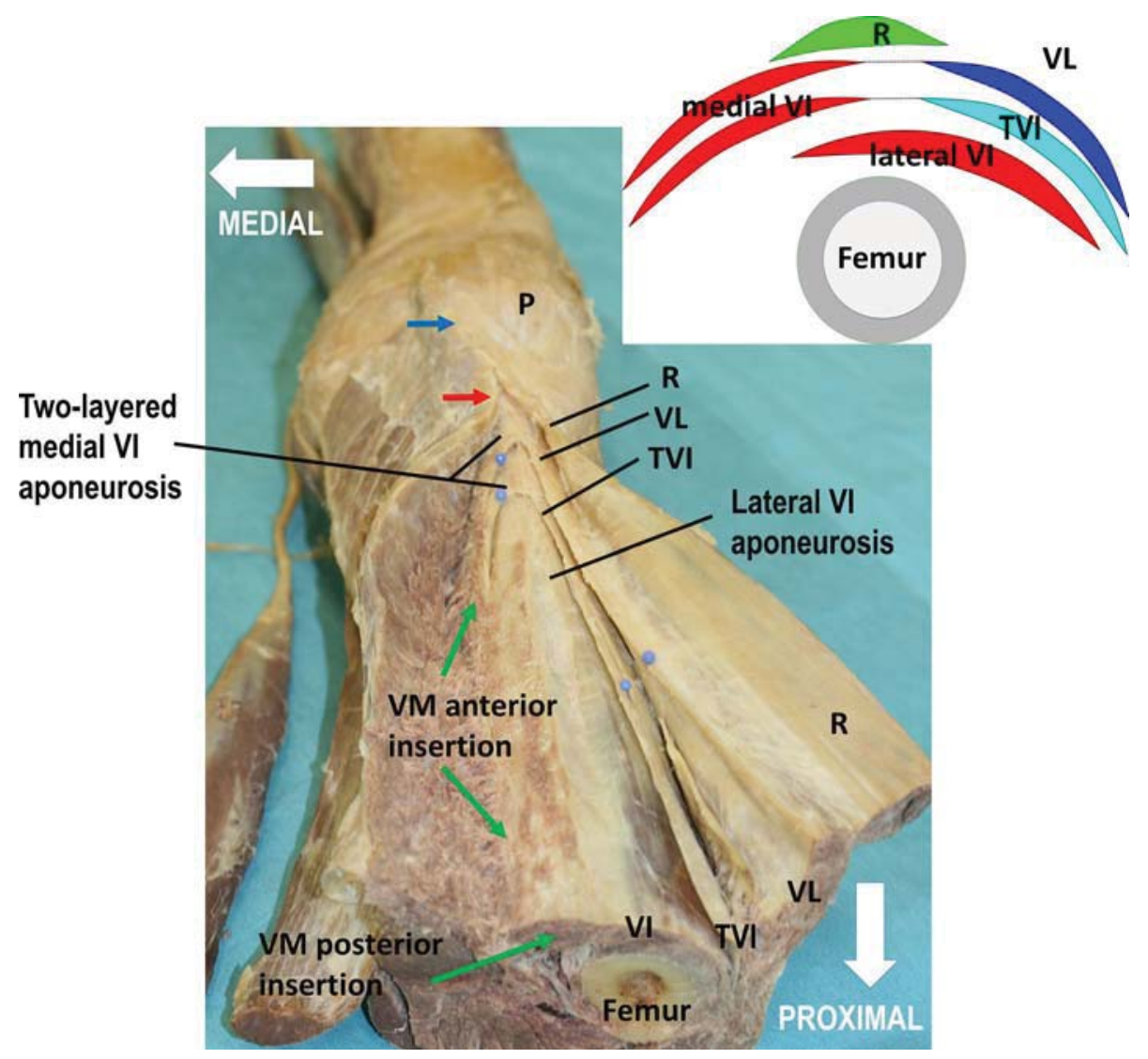

Fig. 3 Insertion of the vastus medialis into the vastus intermedius in relation to the other components and architecture of the extensor apparatus of the knee joint. A cross section of a right thigh with the extensor apparatus of the knee joint is shown (same specimen as in Fig. 2). The vastus medialis (VM) is released from its anterior insertion into the vastus intermedius (VI) and rectus femoris (R), thereby freeing the view to the multi-layered quadriceps tendon. Insertions of the VM into the patella $(\mathrm{P})$, the distal tendon of $\mathrm{R}$ (reflected later-

ally) and the VI are marked with blue, red and green arrows. The VM inserts into the VI aponeurosis on its anterior (VM anterior) and posterior (VM posterior) side. Blue headed needles mark the superficial and deep medial layer of the VI aponeurosis, the tensor vastus intermedius (TVI) and the vastus lateralis (VL). Besides the VM, medial parts of the VI directly contribute to the medial pull on the patella. A corresponding schematic drawing of the three-layered quadriceps tendon is shown at the top right corner

Fig. 4 Schematic drawing of the interaction between the vastus medialis and vastus intermedius. Schematic drawing of interaction between the vastus medialis (VM) and vastus intermedius (VI). The hammock-like origin (grey) and the fleshy clip-type double insertion (red) of VM into the VI aponeurosis (green) is shown. VM cramps the VI aponeurosis like a clip holding a sheet of paper. The medial surface of the femur is bare of muscle fibres (see also Fig. 1). This indicates the function of the medial femur as a gliding surface for the contracting VM muscle units (red)

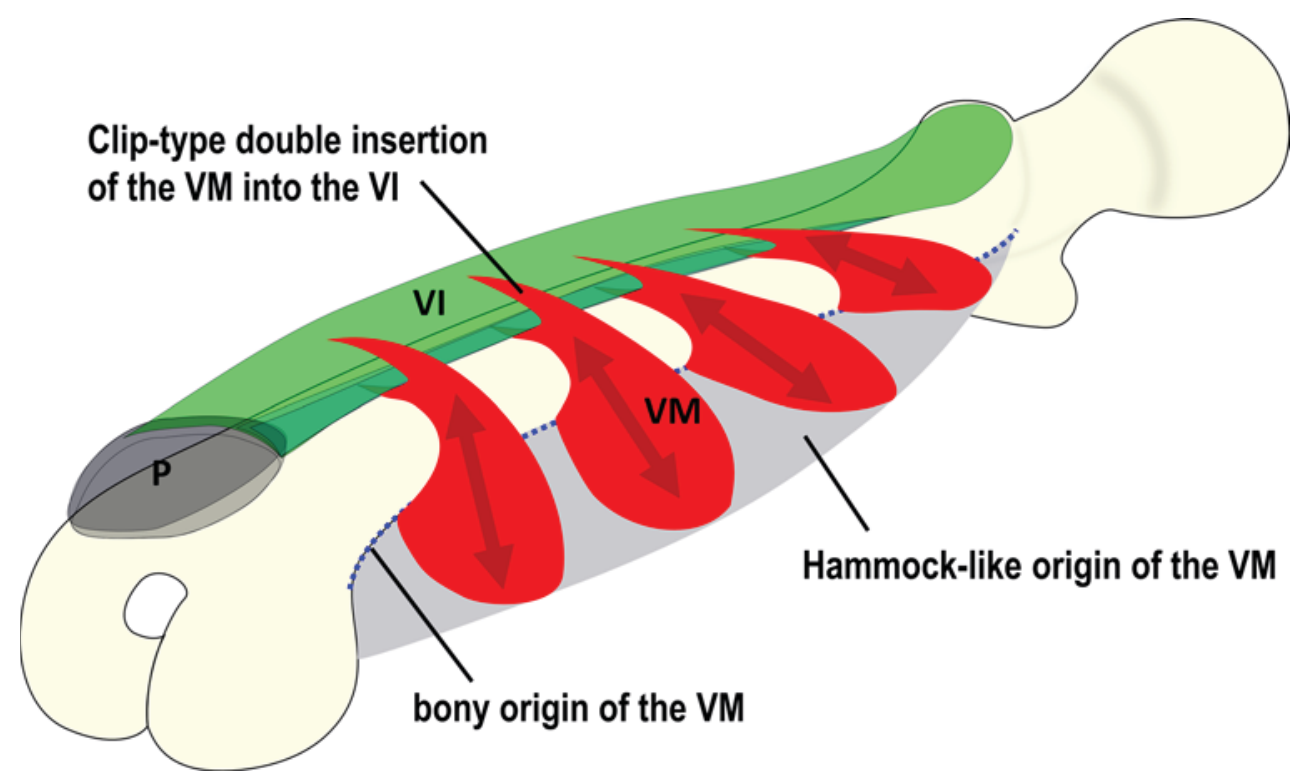


Fig. 5 Nerve supply to the extensor apparatus of the knee joint. The anterior view to a left proximal thigh with the nerve supply to the extensor apparatus is shown. For better visualization of the femoral nerve branches, the sartorius (S) and rectus femoris $(\mathrm{R})$ muscles were transected and elevated. Some nerve branches are underlined with black paper. The red sticker indicates the middle of the neck of femur on the intertrochanteric line. The vastus medialis (VM) is innervated by the medial division of the femoral nerve; this nerve branch to the VM (yellow arrow) runs along the anteromedial border of the muscle. It separates from the saphenous nerve (double arrow) very proximally. Tensor fasciae lata $(\mathrm{T})$, gluteus medius $(\mathrm{G})$, vastus lateralis (VL) and tensor vastus intermedius (TVI) are also labelled
Medial division of femoral nerve

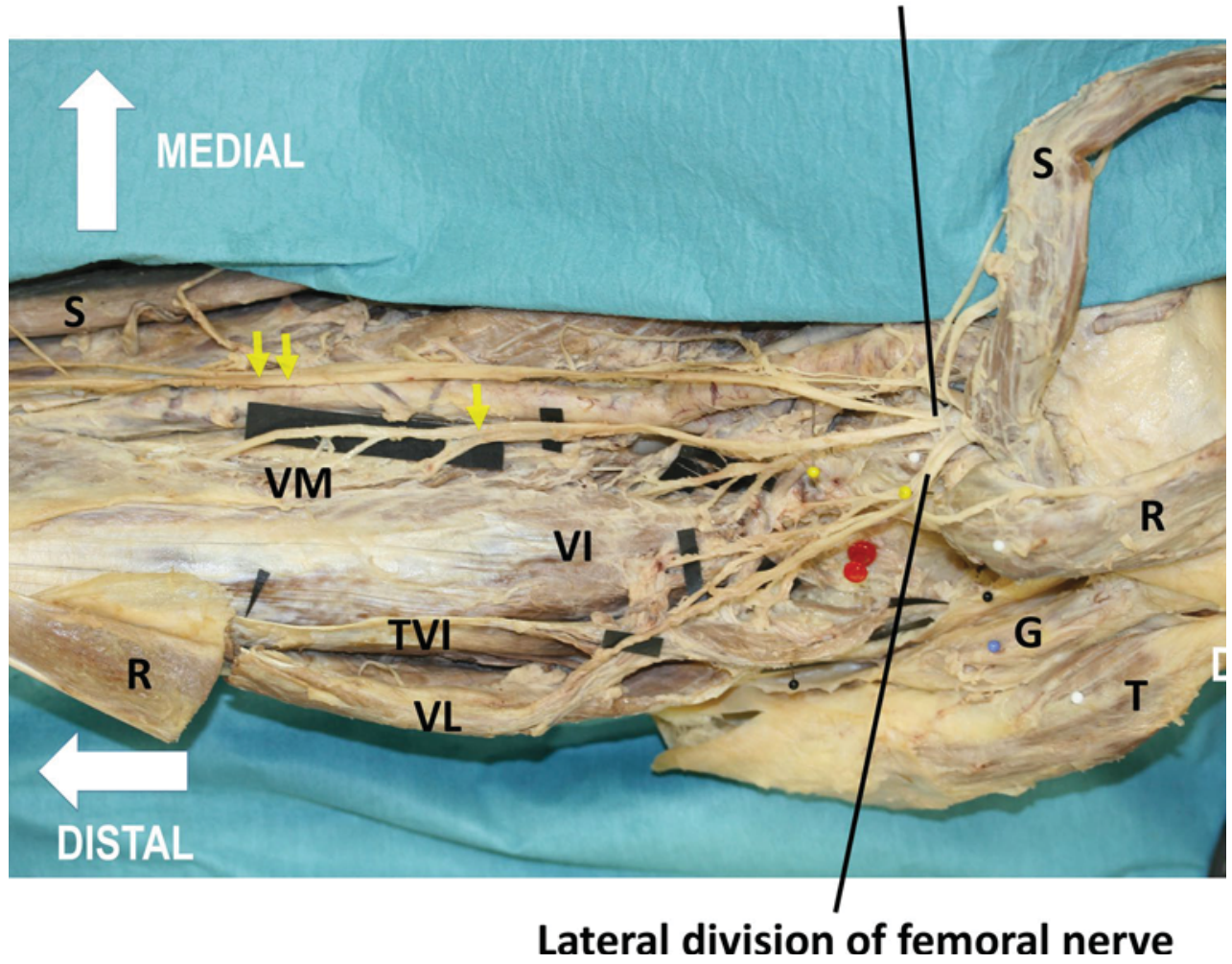

build the superficial part of the intermediate layer of the quadriceps tendon (Fig. 3).

\section{Origin, course and insertion of the vastus intermedius (VI)}

The fibres of the VI arise from the anterior and lateral aspects of the proximal two-thirds of the femoral shaft, including the lateral lip of the linea aspera. Its attachment to the medial femur is restricted to an area proximally and is very close to the intertrochanteric line. The VI is a complex multi-layered structure; the distal two-thirds of the VI is covered by a strong aponeurosis which continues into the quadriceps tendon. The medial part of this VI aponeurosis is divided into a superficial and deep layer (Figs. 2, 3 ) and, together with the lateral part of the VI aponeurosis, provides a wide area for the double insertion of the fleshy fibres of the VM as described above (clip-type double insertion of the VM into the VI) (Figs. 2, 3).

The thickness of the medial part of the VI aponeurosis increases steadily from proximal to distal as more muscle fibre units of the VM insert on both sides. Superficial and deep layers of the medial VI aponeurosis fuse with the aponeuroses of the tensor vastus intermedius and vastus lateralis, respectively (Figs. 2, 3). Together they form the two-layered intermediate layer of the quadriceps tendon. Therefore, the VI contributes firstly to the deep layer of the quadriceps tendon through its lateral part of the VI aponeurosis and secondly to the intermediate layer of the quadriceps tendon by its two-layered medial VI aponeurosis (Figs. 2, 3). In two specimens, some distal fibres of the superficial layer of the medial part of the VI aponeurosis featured a completely separate aponeurosis, which fused with the posterior gliding aponeurosis of the VM. It emerged as the superficial sheet into the middle layer of the quadriceps tendon. Multiple muscle bundles, up to six in number, below the VI aponeurosis continued distally as the articularis genus and did not merge into the quadriceps tendon.

\section{Innervation of VM and VI}

In all specimens, the VM was innervated by the same medial division of the femoral nerve. One long, thick branch coursed distally in a fibrotic tunnel along the anteromedial border of the muscle giving up many side branches to and between the muscle portions of VM (Figs. 1, 5). A second branch, which divided from the same medial division of the femoral nerve, gave branches to the proximal parts of the VM and to the medial parts of the VI (Fig. 5). These branches had a short course and were often hidden by branches of the lateral circumflex femoral artery.

In contrast to the medial part of VI, the lateral part was innervated proximally by multiple short nerve branches 
Fig. 6 Schematic drawings of the extensor apparatus of the knee joint. In these schematic drawings, the blue, red and green dotted lines indicate the insertion sites of the vastus medialis (VM). a As described traditionally in anatomy textbooks and the current literature. Vastus medialis longus (VML) and vastus medialis obliquus (VMO) are labelled. b According to the present study, besides the insertion into the patella (P) (blue) and quadriceps tendon (red), the largest insertion expands over the whole length of the vastus intermedius (VI) aponeurosis (green). This insertion has a fleshy double insertion-a strong and large insertion on the anterior side and a weaker insertion on the posterior side (see also Figs. 2, 3, 4, 7b). Besides the VM, medial parts of the VI insert into the medial aspect of the patella (see also Fig. 3). The VM consists of "multiple muscle units" (*), which are orientated at various angles. Rectus femoris (R), tensor vastus intermedius (TVI) and vastus lateralis (VL) are also labelled
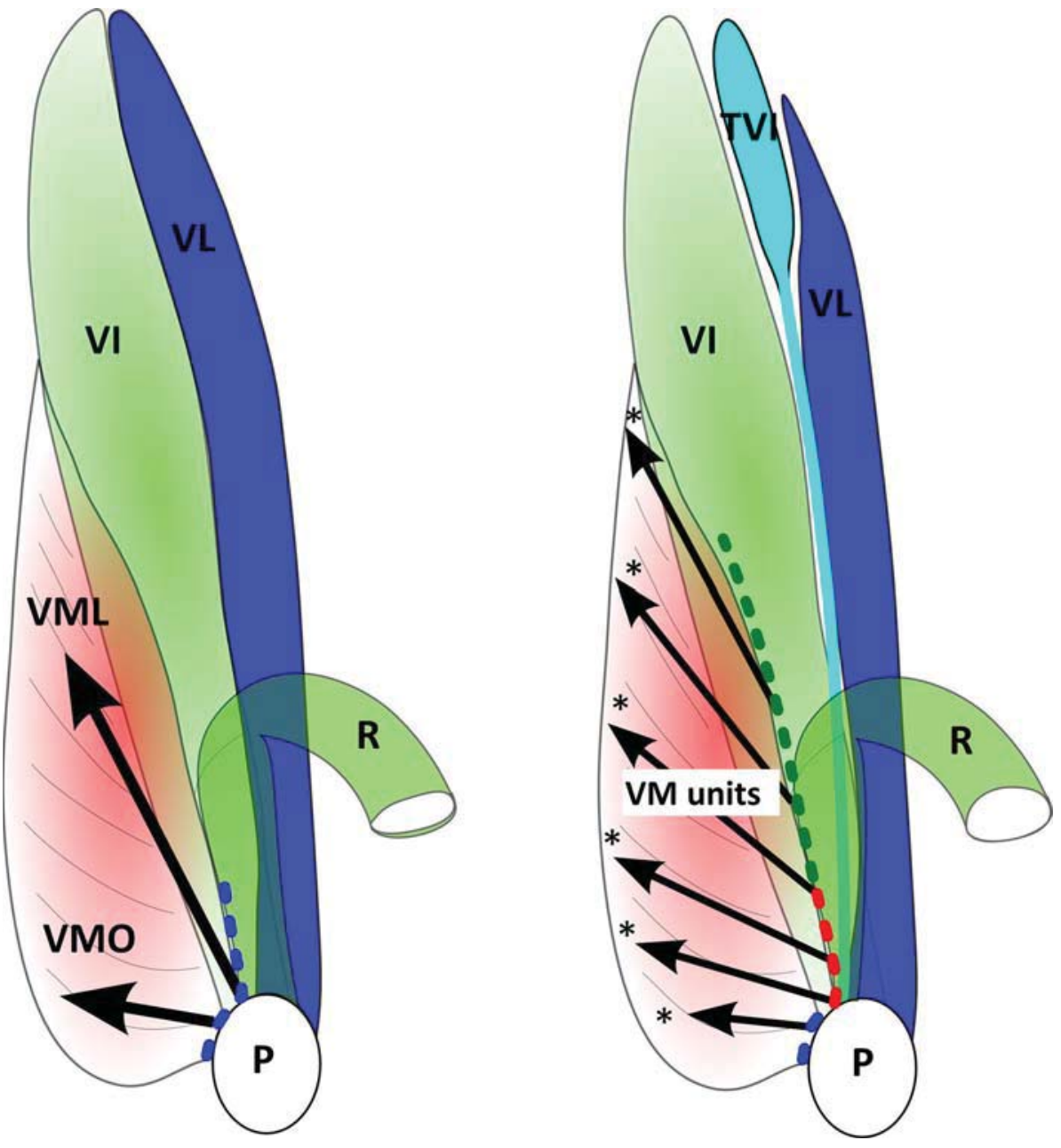

from the lateral division of the femoral nerve. The same division supplied branches to the tensor vastus intermedius (TVI) [10] and the vastus lateralis (Fig. 5).

\section{Discussion}

An important finding of the present study is that the insertion of VM is not limited to the distal end of the quadriceps tendon and the patella, as has been described in many textbooks of anatomy [37, 39, 41, 45]. In contrast, the insertion of the VM expands from the joint capsule and the patella to the medial edge of the rectus femoris and the aponeurosis of the VI (Figs. 2, 3, 6). The fleshy muscle units of the VM hold and pull the VI aponeurosis medially like a clip holding a sheet of paper (Fig. 4). It could be said that the VI aponeurosis is enclosed by the muscle fibres of the VM and, therefore, is located deep to the muscle surface of the VM (Figs. 2, 4). This leads to the impression, as described in the literature, of the existence of an aponeurosis belonging to the VM on its deep surface which is attached to the medial border of the patella $[4,9,23,38,43,52]$. The present dissections revealed that this aponeurosis, which is located in the interior of the VM muscle, in reality belongs to the VI muscle (Figs. 2, 4).

The dilemma about the meaning of this "deep aponeurosis" is highlighted by descriptions in the ancient literature. Gegenbaur [9] stated that in the upper part, VM muscle fibres either merge in a terminal aponeurosis inside the muscle that finally fuse with the lower third of the VI aponeurosis or insert directly in the terminal tendon of the VI [9]. Henle [16] illustrated that medial portions of the extensor cruris (i.e. VM) blend in a vertically oriented aponeurosis that belongs to the vastus anterior (i.e. VI) [16]. An attachment of the VM muscle fibres into the VI has also been mentioned by others [11, 24, 52, 53]. Modern textbooks of anatomy do not show these findings (Fig. 6) [37, 39, 41, 45].

The present results demonstrate that not only the VM, but also substantial parts of the VI insert into the medial aspect of the extensor apparatus of the knee joint (Fig. 3) and, therefore, build strong counterparts to the lateralacting forces on the patella. This functions as a dynamic medial stabilizer of the patella and is supported by the 
existing aponeurotic connections between the VM, VI and the medial patellofemoral ligament [32, 34, 38, 42]-the primary, static, medial restraint of the patella. Together, these structures are activated during contraction of the quadriceps, making the whole system of fundamental importance for the stability of the medial patellofemoral joint [38]. In view of the considerable interaction between VM and VI, the importance of the VM as medial-acting force is by no means restricted to the distal part of its muscle fibres (Figs. 4, 6). These findings are in contrast to previous reports [18, 25, 26, 34].

These investigations revealed that the patellar insertion of VM is very small compared to the distinct attachments into the rectus femoris and, above all, into the aponeurosis of the VI (Figs. 2, 3, 4, 6). Thus, the angle of the distal fibres, which directly insert into the medial aspect of the patella, does not place the muscle in a position to act as a strong medial stabilizer [26, 30, 31, 37].

With regard to the orientation of its muscle fibres, the direct force supplied by VM for extension of the knee joint is limited [26]. In an in vitro study, Sakai et al. [44] studied the influence of weakness of the distal part of the VM muscle on patellar tracking and reported that simulated VM pull had little influence on patella tracking. Their finding suggested that another factor must regulate or resist the lateral displacement of the patella (Fig. 3). A biomechanical study of the quadriceps using amputated specimens to test the extensor capacity of the different components of this muscle showed that all the long components were able to extend the knee fully. However, the knee could not be extended by the oblique fibres of the VM [26].

Little attention has been given to the proximal part of the VM, in particular, its interaction with the VI. Most investigations deal primarily with the distal part of the VM, which is also termed vastus medialis obliquus (VMO) (Fig. 6) [3, $14,26,40,46,48,50,53]$. We were not able to find a separate innervation to the different sections of VM, nor a clearly distinct fascial plane between the two heads. All the muscle fibres of the VM were supplied by the same medial division of the femoral nerve (Fig. 5). There is evidence that the more distal fibres, because of their lever arm arrangement, help to maintain patellar alignment. However, the crosssectional area of the proximal VM, where it inserts into the aponeurosis of the VI, is much larger and could, therefore, contribute as a strong stabilizer against the lateral patellar forces. We propose that the VM consists of multiple muscle units, with each muscle unit having its specific orientation. The VM inserts through the VI along the longitudinal components of the extensor apparatus at three sections (Figs. 4, 6). Based on these findings, the VM exerts a predominantly medial pull on the longitudinal component of the quadriceps muscle group (“medial pull-mechanism”).
Patients with lesions that affect the knee commonly demonstrate visible atrophy of the VM prior to causing any measurable decrease in the circumference of the thigh [26]. These patients also lack active full extension. This supports the assertion by many authors and clinicians that the VM is mainly active during the terminal range of extension, and many exercise programmes to strengthen the knee extensor muscles are based on this interpretation. However, there is continued activation of VM throughout the range of motion from 0 to $90^{\circ}$ of flexion. The function of the interacting VM and VI could also be the reason why electromyography (EMG) studies showed continuous activation of the VM during the entire gait cycle [15, 26, 27, 38, 47, 51]. Physical therapists often focus their therapy on enhancement of VM activity over the rest of quadriceps muscles; however, this is difficult to achieve [49]. EMG studies have shown that all components of the quadriceps act in conjunction and that none of the components are predominantly responsible for fully extending the knee $[26,51]$.

The EMG observations in the literature, together with findings of the present anatomical study, suggest that the VM acts as an indirect extensor at the knee. It can be hypothesized that by pulling the longitudinal components of VI and rectus femoris medially and dorsally, the VM tightens and shortens these quadriceps muscles, much like a belt around the waist. Obviously, this shortening of the length of the quadriceps (indirect extensor mechanism) is most important during the terminal phase of extension.

It has been shown that nearly twice as much force is needed by the quadriceps group to produce full extension of the knee in comparison with extending the knee to only $15^{\circ}$ of flexion [26]. This explains the importance of the $\mathrm{VM}$ in the terminal range of extension where it is mostly active. The VM triggers the longitudinal components of the quadriceps muscle group by creating a pre-strain at the terminal range of extension. Without this "shortening and pre-straining mechanism" in the terminal phase of extension, the quadriceps muscles would be too long and not sufficiently powerful to effect full extension of the knee joint. In other words, the required orchestral shortening of the quadriceps muscle group for full knee extension could not be achieved by contraction of the muscle fibres (direct extensor mechanism) alone. A simplified model of this mechanism is shown in. Fig. 7 (see supplementary video clip)

Figure. 7 Conversely, the length of the quadriceps muscle group has to extend maximally to create full flexion at the knee. Including the VM, the quadriceps muscle group features an extraordinary mechanism to adjust its length during the entire range of motion. Therefore, any dysfunction of the VM would automatically influence the whole range of motion of the knee joint, which can be observed 

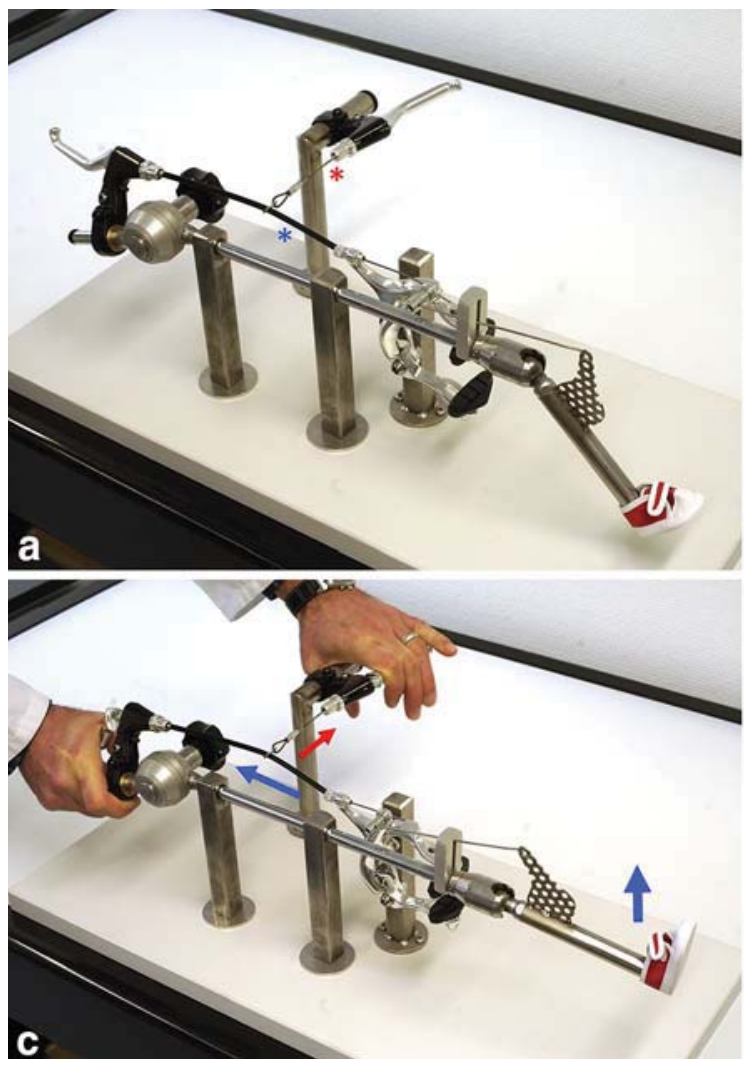

Fig. 7 Hypothetic simplified functional model of the extensor apparatus of the knee joint based on the present anatomical findings. a In a simplified example, the extensor apparatus of the knee joint works like the conventional brake of a bicycle. b Unlike braking, however, the action of the extensor apparatus of the knee joint leads to extension of the knee joint. c A side pull (red arrow) on the longitudinal cord that connects the braking handle with brake shoes would lead to a pretension of the pulling mechanism and consequently to a more efficient braking action. In other words, the braking action can be

in daily clinical practice. The regulative function of the VM during the entire range of motion of the knee joint is indispensable and explains why the VM, of all the quadriceps components, atrophies most rapidly due to inactivity, loss of knee extension after trauma or surgery, or after effusion in the joint. Similarly, failure of the VM can lead to restrictions in knee joint range of motion.

The medial surface of the femur is, in contrast to some descriptions in the literature $[26,36,54,55] *$ bare of muscle attachments. This indicates its function as a gliding surface for the mediodorsally contracting VM muscle units (Figs. 1, 4).

The results of this study should also influence the radiological interpretation of MRI transections of the extensor apparatus (Fig. 8). The medial aponeurosis of the VI, traditionally regarded as an intermuscular septum or space between the VI and VM [2, 6, 36, 56], does not correspond to the intermuscular plane between these two muscles

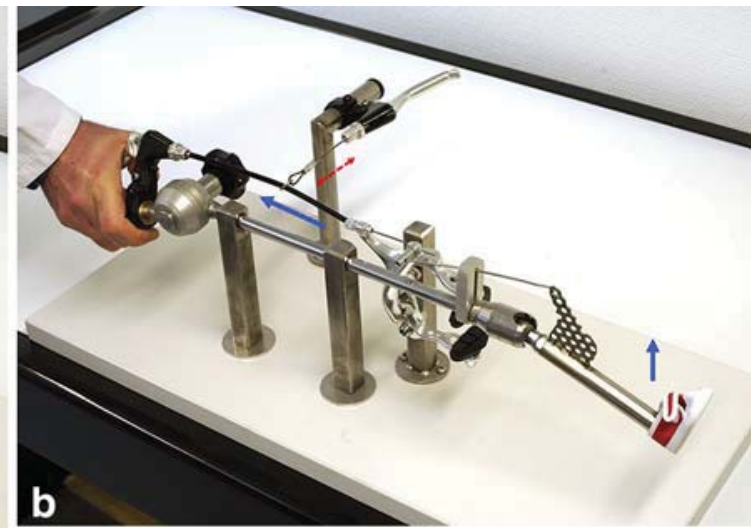

controlled by the side pull (red arrow) on the cord. With regard to the extensor mechanism of the knee joint, this control function is adopted by the vastus medialis (VM) muscle. Due to its medial and dorsal pull (red arrow) on the longitudinal components of the quadriceps muscle group, the VM influences the length, effectiveness and power of the extensor apparatus of the knee joint. This shortening of the quadriceps muscle group is most important during the terminal range of extension c. Thus, the VM regulates the extensor apparatus during the entire range of knee joint motion.

(Fig. 7). This is due to the double attachment of the VM into the VI aponeurosis (the clip-type double insertion shown in Figs. 2, 4). The VM and VI form a functional connection. This interpretation is supported by the innervation pattern of both muscles (Fig. 5). With regard to its innervation, the VI can also be divided into a medial and lateral section [10-13].

The functions of the VM can be summarized as follows:

1. The VM is an effective indirect extensor of the knee joint, mainly important during the terminal phase of extension (Pretension).

2. The VM adjusts the length of the quadriceps muscle group and regulates the knee joint during the entire range of motion.

3. VM (together with the VI) represents a dynamic restraint to lateral tracking of the patella. 


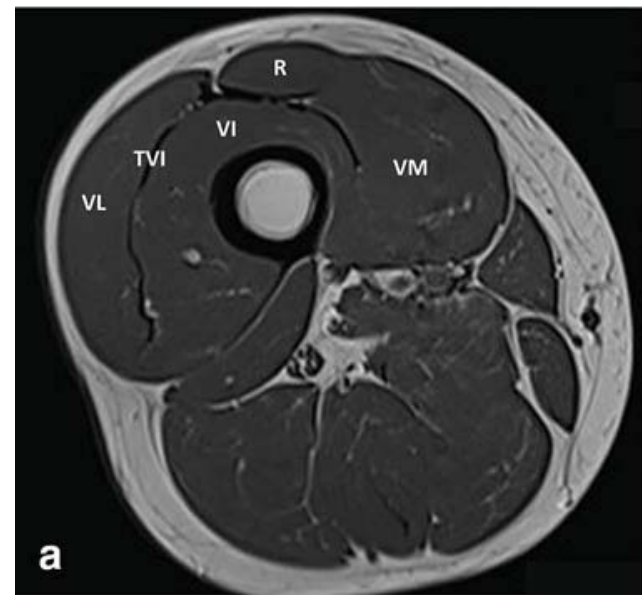

Fig. 8 MRI cross section of the extensor apparatus of the knee joint. a MRI cross section through the middle third of the right thigh (marked with colour) is shown. b The same cross section as Fig. 7a but marked with colour. The aponeurosis of the vastus intermedius (VI) is labelled green, and the tensor vastus intermedius (TVI) blue. Vastus lateralis (VL) is also labelled. Vastus medialis (VM) inserts into the VI aponeurosis (green) on its anterior and posterior side (*),

Figure. 8 Most modern anatomy textbooks do not reflect the complex anatomy and interaction between the different muscle components of the quadriceps group. Classical anatomy has defined each muscle as a separate entity with a unique function at the joint it spans, so it is common to view muscles as mechanically independent actuators. As a result, many musculoskeletal models have been developed based on a simplified view of the human quadriceps anatomy [7, $22,35,47]$. However, in the last decade, the potential of force transmission between synergistic skeletal muscles through connective tissue linkage has been demonstrated [20, 29, 57].

The interaction between the VM and VI, with its responsibility for the extension of the knee joint and influence on the patellofemoral function, leads to a ready understanding of common clinical problems found at the knee joint as the body attempts to meet contradictory demands for both mobility and stability. The results of this study have many implications including the understanding and treatment of patellofemoral instability, therapy for knee malfunction or knee stiffness after trauma or surgery, the choice of surgical approach to the femur and knee joint, treatment of the extensor apparatus in knee revision settings and radiological interpretation of MRI transections of the extensor apparatus.

The present study has few limitations, in which one important limitation is that the quadriceps tendon was investigated in embalmed cadaveric specimens from elderly donors. Age-related muscle atrophy may well have distorted some results. In addition, embalmed tissue has been reported to shrink by between 2 and $12 \%$ [5, 8]. This could

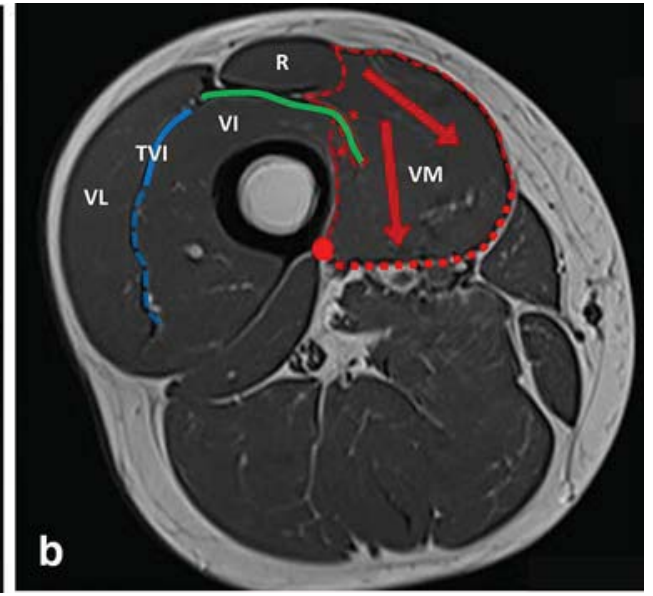

but also into the rectus femoris muscle $(\mathrm{R})$. The thick red dotted line corresponds to the hammock-like origin of the medial intermuscular septum and medial lip of the linea aspera (red dot). The red arrows indicate the direction of VM pull on the longitudinal components of the extensor apparatus of the knee joint (from anterior, lateral and distal to medial, dorsal and proximal)

have affected absolute values for variables such as measured distances. Nevertheless, the fundamental architecture of the quadriceps muscle group is likely to have been preserved. Considering the complexity of the extensor apparatus of the knee joint, further investigation of its morphology in healthy young individuals is warranted.

\section{Conclusion}

Comprehension of the function and architecture of the quadriceps muscle group is strongly linked with an understanding of the anatomy of the VM and VI. VM consists of multiple muscle units inserting into the entire VI aponeurosis. Together, VM and VI build a potential functional muscular complex. From an anatomical point of view, it can be suggested that the VM acts as an indirect extensor of the knee joint, regulating and adjusting the length of the extensor apparatus throughout the entire range of motion. It is of clinical importance that besides the VM, substantial parts of the VI directly contribute to the medial pull on the patella and help to maintain medial tracking of the patella during knee extension. Surgery or trauma in the anteromedial aspect of the quadriceps muscle group might alter a delicate interplay between the VM and VI, which would affect the extensor apparatus as a whole.

Authors' contribution Grob K (GK), Manestar M (MM), Filgueira L (FL), Kuster MS (KMS), Gilbey H (GH), Ackland T (AT). GK carried out the anatomical dissections and took the photographs. GK drafted the manuscript. MM and FL participated in the design of the study and the anatomical dissections. MM and KMS also participated in the 
literature research. GH has been involved first in the drafting the manuscript and second in the revision process. AT and KMS gave the final approval of the version to be published. All authors were involved in the interpretation of the results of the anatomical dissections.

\section{Compliance with ethical standards}

Conflict of interest The authors declare that they have no conflict of interest.

\section{Funding None.}

Ethical approval This article does not contain any studies with human participants or animals performed by any of the authors.

Informed consent In this article, no patient care was involved.

\section{References}

1. Blazevich AJ, Gill ND, Zhou S (2006) Intra- and intermuscular variation in human quadriceps femoris architecture assessed in vivo. J Anat 209:289-310

2. Blemker SS, Delp SL (2006) Rectus femoris and vastus intermedius fiber excursions predicted by three-dimensional muscle models. J Biomech 39:1383-1391

3. Bose K, Kanagasuntheram R, Osman MB (1980) Vastus medialis oblique: an anatomic and physiologic study. Orthopedics 3:880-883

4. Bryce TH (1923) Myology. In: Quain's elements of anatomy, 11th ed. vol 4. pp 236-241

5. Cutts A (1988) Shrinkage of muscle fibres during the fixation of cadaveric tissue. J Anat 160:75-78

6. Engstrom CM, Loeb GE, Reid JG, Forrest WJ, Avruch L (1991) Morphometry of the human thigh muscles. A comparison between anatomical sections and computer tomographic and magnetic resonance images. J Anat 176:139-156

7. Erdemir A, McLean S, Herzog W, van den Bogert AJ (2007) Model-based estimation of muscle forces exerted during movements. Clin Biomech (Bristol, Avon) 22:131-154

8. Friederich JA, Brand RA (1990) Muscle fiber architecture in the human lower limb. J Biomech 23:91-95

9. Gegenbaur C (1899) Lehrbuch der Anatomie des Menschen. Verlag von Wilhelm Engelmann, Leibzig, p 449

10. Grob K, Ackland T, Kuster M, Manestar M, Filgueira L (2016) A newly discovered muscle: the tensor of the vastus intermedius. Clin Anat 29:256-263

11. Grob K, Manestar M, Filgueira L, Ackland T, Gilbey H, Kuster MS (2016) New insight in the architecture of the quadriceps tendon. J Exp Orthop 3:32

12. Grob K, Monahan R, Gilbey H, Ackland T, Kuster MS (2015) Limitations of the vastus lateralis muscle as a substitute for lost abductor muscle function: an anatomical study. J Arthroplasty 30:2338-2342

13. Grob K, Monahan R, Gilbey H, Yap F, Filgueira L, Kuster M (2015) Distal extension of the direct anterior approach to the hip poses risk to neurovascular structures: an anatomical study. J Bone Joint Surg Am 97:126-132

14. Günal I, Araç S, Sahinoğlu K, Birvar K (1992) The innervation of vastus medialis obliquus. J Bone Joint Surg Br 74:624

15. Hallén LG, Lindahl O (1967) Muscle function in knee extension. An EMG study. Acta Orthop Scand 38:434-444
16. Henle J (1855) Handbuch der Systematischen Anatomie des Menschen. In drei Bänden, Erster Band. Erste Abteilung. Knochenlehre. Braunschweig, Druck und Verlag von Friedrich Vieweg und Sohn. 255-260

17. Hollinshead W, Rosse C (1985) Textbook of anatomy. Harper (ed) Philadelphia

18. Holmes SW, Clancy WG (1998) Clinical classification of patellofemoral pain and dysfunction. J Orthop Sports Phys Ther 28:299-306

19. Hubbard JK, Sampson HW, Elledge JR (1997) Prevalence and morphology of the vastus medialis oblique muscle in human cadavers. Anat Rec 249:135-142

20. Huijing PA (2009) Epimuscular myofascial force transmission: a historical review and implications for new research. International society of biomechanics muybridge award lecture, Taipei, 2007. J Biomech 42:9-21

21. Hyong IH, Kang JH (2013) Activities of the vastus lateralis and vastus medialis oblique muscles during squats on different surfaces. J Phys Ther Sci 25:915-917

22. Klein Horsman MD, Koopman HFJM, van der Helm FCT, Prosé LP, Veeger HEJ (2007) Morphological muscle and joint parameters for musculoskeletal modelling of the lower extremity. Clin Biomech (Bristol, Avon) 22:239-247

23. LT (1893) D'Anatomie humaine. In: Doin O (ed) Traité, Paris, pp $170-175$

24. Last RJ (1952) On the form and structure of muscles. J Bone Joint Surg Br 34-B:295-305

25. Lefebvre R, Leroux A, Poumarat G, Galtier B, Guillot M, Vanneuville G, Boucher JP (2006) Vastus medialis: anatomical and functional considerations and implications based upon human and cadaveric studies. J Manip Physiol Ther 29:139-144

26. Lieb FJ, Perry J (1968) Quadriceps function. An anatomical and mechanical study using amputated limbs. J Bone Joint Surg Am 50:1535-1548

27. Lieb FJ, Perry J (1971) Quadriceps function. An electromyographic study under isometric conditions. J Bone Joint Surg Am 53:749-758

28. Lin Y-F, Lin J-J, Jan M-H, Wei T-C, Shih H-Y, Cheng C-K (2008) Role of the vastus medialis obliquus in repositioning the patella: a dynamic computed tomography study. Am J Sports Med 36:741-746

29. Maas H, Sandercock TG (2010) Force transmission between synergistic skeletal muscles through connective tissue linkages. J Biomed Biotechnol 2010:575672

30. Malone T, Davies G, Walsh WM (2002) Muscular control of the patella. Clin Sports Med 21:349-362

31. McConnell J (2002) The physical therapist's approach to patellofemoral disorders. Clin Sports Med 21:363-387

32. Mochizuki T, Nimura A, Tateishi T, Yamaguchi K, Muneta T, Akita K (2013) Anatomic study of the attachment of the medial patellofemoral ligament and its characteristic relationships to the vastus intermedius. Knee Surg Sports Traumatol Arthrosc 21:305-310

33. Nozic M, Mitchell J, de Klerk D (1997) A comparison of the proximal and distal parts of the vastus medialis muscle. Aust $\mathrm{J}$ Physiother 43:277-281

34. Panagiotopoulos E, Strzelczyk P, Herrmann M, Scuderi G (2006) Cadaveric study on static medial patellar stabilizers: the dynamizing role of the vastus medialis obliquus on medial patellofemoral ligament. Knee Surg Sports Traumatol Arthrosc 14:7-12

35. Pandy MG (2001) Computer modeling and simulation of human movement. Annu Rev Biomed Eng 3:245-273

36. Pasta G, Nanni G, Molini L, Bianchi S (2010) Sonography of the quadriceps muscle: examination technique, normal anatomy, and traumatic lesions. J Ultrasound 13:76-84 
37. Peeler J, Cooper J, Porter MM, Thliveris JA, Anderson JE (2005) Structural parameters of the vastus medialis muscle. Clin Anat 18:281-289

38. Placella G, Tei MM, Sebastiani E, Criscenti G, Speziali A, Mazzola C, Georgoulis A, Cerulli G (2014) Shape and size of the medial patellofemoral ligament for the best surgical reconstruction: a human cadaveric study. Knee Surg Sports Traumatol Arthrosc 22:2327-2333

39. Platzer W (2013) Taschenatlas anatomie. Bewegungsapparat. 11th ed. Thieme, Stuttgart, New York. pp 248-249

40. Pocock GS (1963) Electromyographic study of the quadriceps during resistive exercise. J Am Phys Ther Assoc 43:427-434

41. Putz R, Papst R (2011) Sobotta atlas of human anatomy. In: Elsvier/Urban \& Fischer (ed). 15th ed. Munich. pp 302-304

42. Reider B, Marshall JL, Koslin B, Ring B, Girgis FG (1981) The anterior aspect of the knee joint. J Bone Joint Surg Am 63:351-356

43. Drake RL, Vogl AW (2015) Gray's Anatomy for students. 3rd ed. Churchill Livingstone Elsevier pp 592-594

44. Sakai N, Luo ZP, Rand JA, An KN (2000) The influence of weakness in the vastus medialis oblique muscle on the patellofemoral joint: an in vitro biomechanical study. Clin Biomech (Bristol, Avon) 15:335-339

45. Schünke M, Schulte E, Schuhmacher U (2011) Prometheus LernAtlas der Anatomie: Allgemeine Anatomie und Bewegungssystem. Thieme, Stuttgart

46. Schünke M, Schulte E, Schumacher U (2011) In: Schünke M (ed) Prometeus Lernatlas der Anatomie: Allgemeine Anatomie und Bewegungssystem. Georg Thieme Verlag, Stuttgart

47. Shelburne KB, Pandy MG (1997) A musculoskeletal model of the knee for evaluating ligament forces during isometric contractions. J Biomech 30:163-176
48. Skinner EJ, Adds PJ (2012) Vastus medialis: a reappraisal of VMO and VML. J Phys Ther Sci 24:475-479

49. Smith TO, Bowyer D, Dixon J, Stephenson R, Chester R, Donell ST (2009) Can vastus medialis oblique be preferentially activated? A systematic review of electromyographic studies. Physiother Theory Pract 25:69-98

50. Smith TO, Nichols R, Harle D, Donell ST (2009) Do the vastus medialis obliquus and vastus medialis longus really exist? A systematic review. Clin Anat 22:183-199

51. Speakman HG, Weisberg J (1977) The vastus medialis controversy. Physiotherapy 63:249-254

52. Thiranagama R (1990) Nerve supply of the human vastus medialis muscle. J Anat 170:193-198

53. Toumi H, Poumarat G, Benjamin M, Best TM, Best T, F'Guyer S, Fairclough J (2007) New insights into the function of the vastus medialis with clinical implications. Med Sci Sports Exerc 39:1153-1159

54. Weinstabl R, Scharf W, Firbas W (1989) The extensor apparatus of the knee joint and its peripheral vasti: anatomic investigation and clinical relevance. Surg Radiol Anat 11:17-22

55. Willan PL, Mahon M, Golland JA (1990) Morphological variations of the human vastus lateralis muscle. J Anat 168:235-239

56. Willan PLT, Ransome JA, Mahon M (2002) Variability in human quadriceps muscles: quantitative study and review of clinical literature. Clin Anat 15:116-128

57. Yucesoy CA, Baan GC, Koopman BHFJM, Grootenboer HJ, Huijing PA (2005) Pre-strained epimuscular connections cause muscular myofascial force transmission to affect properties of synergistic EHL and EDL muscles of the rat. J Biomech Eng $127: 819-828$ 